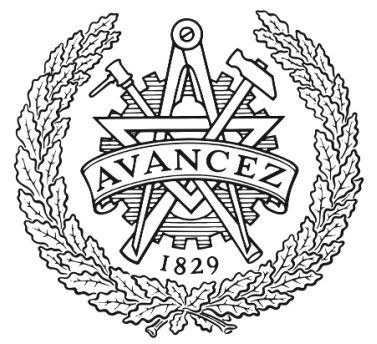

CHALMERS

UNIVERSITY OF TECHNOLOGY

\title{
Nonconjugated Terpolymer Acceptors with Two Different Fused-Ring Electron-Deficient Building Blocks for Efficient All-Polymer Solar Cells
}

Downloaded from: https://research.chalmers.se, 2023-04-26 12:19 UTC

Citation for the original published paper (version of record):

Su, W., Fan, Q., Jalan, I. et al (2021). Nonconjugated Terpolymer Acceptors with Two Different Fused-Ring Electron-Deficient Building Blocks for Efficient All-Polymer Solar Cells. ACS Applied Materials \& Interfaces, 13(5): 6442-6449. http://dx.doi.org/10.1021/acsami.0c17722

N.B. When citing this work, cite the original published paper. 


\section{Nonconjugated Terpolymer Acceptors with Two Different Fused- Ring Electron-Deficient Building Blocks for Efficient All-Polymer Solar Cells}

Wenyan Su, Qunping Fan,* Ishita Jalan, Yufei Wang, Wenhong Peng, Tao Guo, Weiguo Zhu, Donghong $\mathrm{Yu}$, Lintao Hou,* Ellen Moons, ${ }^{*}$ and Ergang Wang*

Cite This: ACS Appl. Mater. Interfaces 2021, 13, 6442-6449

Read Online

ACCESS | Llll Metrics \& More | 回 Article Recommendations | st Supporting Information

ABSTRACT: The ternary polymerization strategy of incorporating different donor and acceptor units forming terpolymers as photovoltaic materials has been proven advantageous in improving power conversion efficiencies (PCEs) of polymer solar cells (PSCs). Herein, a series of low band gap nonconjugated terpolymer acceptors based on two different fused-ring electron-deficient building blocks (IDIC16 and ITIC) with adjustable photoelectric properties were developed. As the third component, ITIC building blocks with a larger $\pi$-conjugation structure, shorter solubilizing side chains, and red-shifted absorption spectrum were incorporated into an IDIC16-based nonconjugated copolymer acceptor PF1-TS4, which built up the terpolymers with two conjugated building blocks linked by flexible thioalkyl chain-thiophene segments. With the increasing ITIC content, terpolymers show gradually broadened

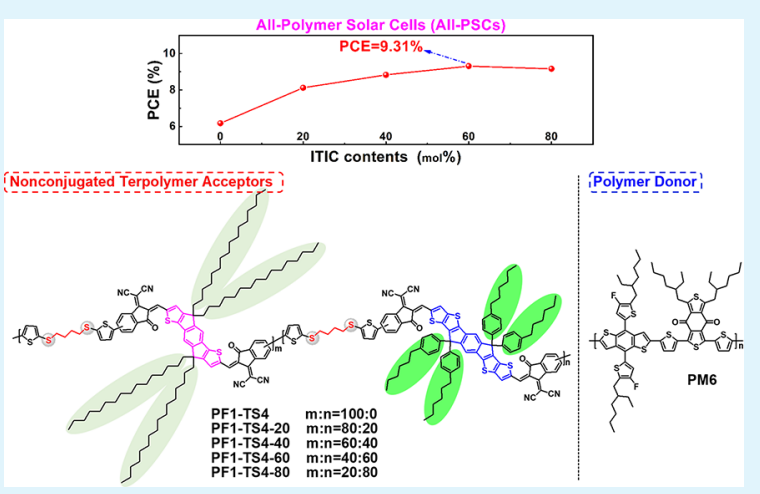
absorption spectra and slightly down-shifted lowest unoccupied molecular orbital levels. The active layer based on terpolymer PF1-TS4-60 with a 60\% ITIC unit presents more balanced hole and electron mobilities, higher photoluminescence quenching efficiency, and improved morphology compared to those based on PF1-TS4. In all-polymer solar cells (all-PSCs), PF1-TS4-60, matched with a wide band gap polymer donor PM6, achieved a similar open-circuit voltage $\left(V_{o c}\right)$ of $0.99 \mathrm{~V}$, a dramatically increased short-circuit current density $\left(J_{\mathrm{sc}}\right)$ of $15.30 \mathrm{~mA} \mathrm{~cm}{ }^{-2}$, and fill factor $(\mathrm{FF})$ of $61.4 \%$ compared to PF1-TS4 $\left(V_{\mathrm{oc}}=0.99 \mathrm{~V}, J_{\mathrm{sc}}=11.21\right.$ $\mathrm{mA} \mathrm{cm}{ }^{-2}$, and $\left.\mathrm{FF}=55.6 \%\right)$. As a result, the PF1-TS4-60-based all-PSCs achieved a PCE of $9.31 \%$, which is $\sim 50 \%$ higher than the PF1-TS4-based ones (6.17\%). The results demonstrate a promising approach to develop high-performance nonconjugated terpolymer acceptors for efficient all-PSCs by means of ternary polymerization using two different A-D-A-structured fused-ring electron-deficient building blocks.

KEYWORDS: all-polymer solar cells, copolymer, nonconjugated polymer acceptor, power conversion efficiency, terpolymer

\section{INTRODUCTION}

During the past 5 years, progress in polymer solar cells (PSCs), with their merits of light weight, low cost, semitransparency, and flexibility, has been dominated by the development of polymer donors and A-D-A-structured fused-ring smallmolecule acceptors (SMAs). ${ }^{1,2}$ This rational molecular design of active layer materials and systematic processing and engineering of devices has led to the state-of-the-art power conversion efficiencies (PCEs) exceeding $17 \%$ so far. $^{3-10}$ Compared to the fused-ring SMA-based systems, all-polymer solar cells (all-PSCs) composed of polymer donor and polymer acceptor materials have some special advantages, such as excellent morphological stability and mechanical properties, which can cater to the requirements of practical application of flexible devices fabricated by roll-to-roll printing techniques. ${ }^{11-14}$ Mainly because of the lack of high-performance polymer acceptors, progress toward efficient all-PSCs has been severely constrained, and their corresponding PCEs still lag behind those of fused-ring SMA-based devices. Tracing the roadmap of PCEs, only a few polymer acceptors have achieved PCEs over $9 \%$ in all-PSCs, with such limited structural electron-deficient units as perylene-diimide, ${ }^{15}$ naphthalene diimide, ${ }^{16-19}$ thiophene-fused diimide, ${ }^{20}$ and $\mathrm{B} \rightarrow \mathrm{N}$-bridged building blocks. ${ }^{21,22}$ However, the low absorption coefficients $\left(\sim 10^{4} \mathrm{~cm}^{-1}\right)$ of these polymer acceptors limit their shortcircuit current density $\left(J_{\text {sc }}\right)$ and PCEs in all-PSCs.

Received: October 2, 2020

Accepted: January 12, 2021

Published: January 26, 2021 
Scheme 1. Molecular Structures of (a) Nonconjugated Copolymer Acceptor PF1-TS4 and Terpolymer Acceptors PF1-TS4-xx with Different IDIC16/ITIC Ratios and (b) the Polymer Donor PM6

(a) Nonconjugated Terpolymer Acceptors

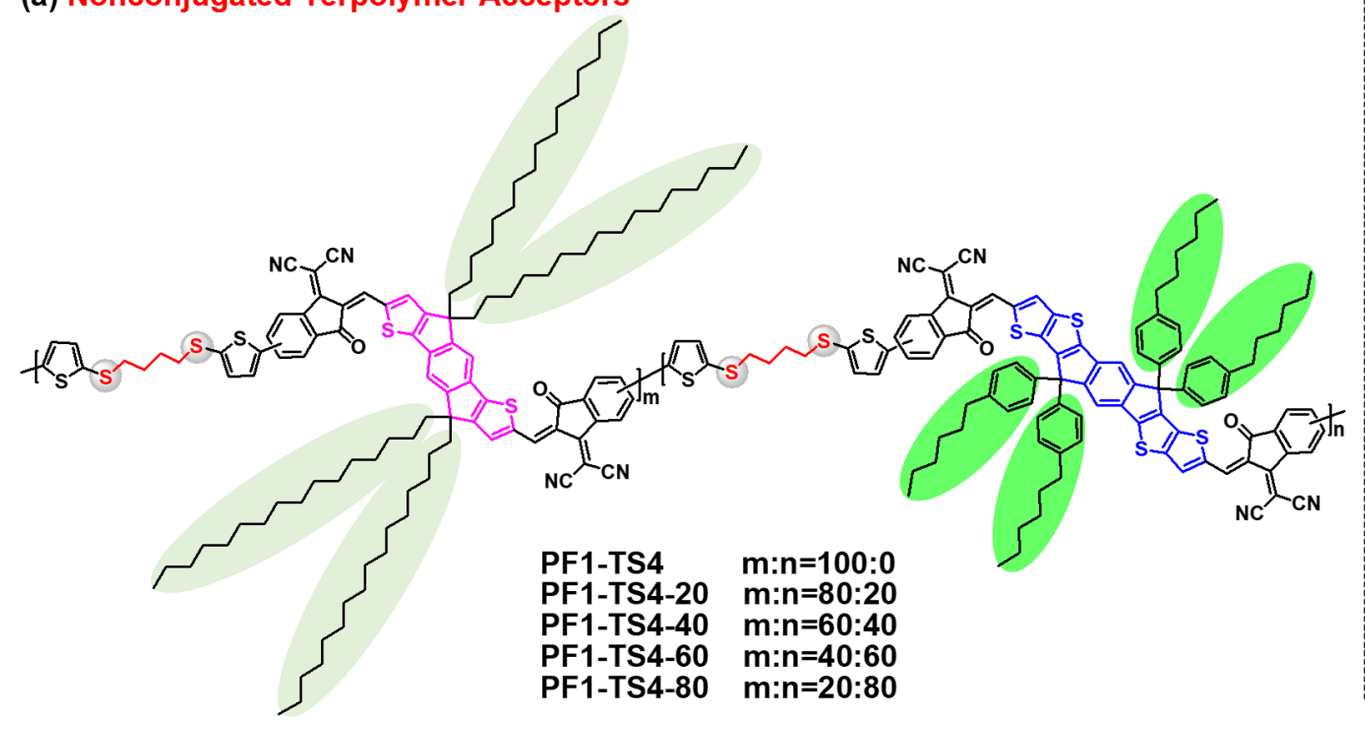

(b) Polymer Donor

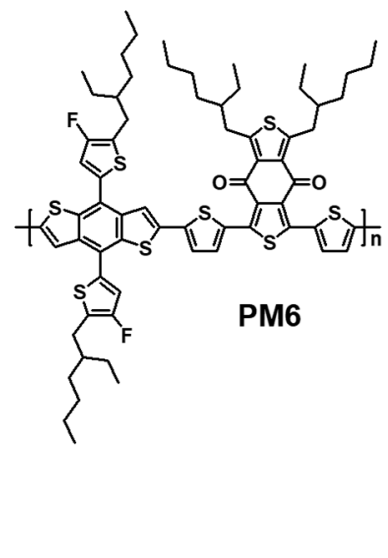

Recently, in the search for polymer acceptors with improved absorption properties, a novel polymer acceptor PZ1 with a low band gap of $1.55 \mathrm{eV}$ and a high maximum absorption coefficient $\left(>10^{5} \mathrm{~cm}^{-1}\right)$ was successfully synthesized by Li et al. by polymerizing a large $\pi$-fused-ring SMA building block (IDIC16) with long solubilizing sidechains and achieved an impressive PCE of $9.19 \%$ in all-PSCs. ${ }^{23}$ Subsequently, some derivatives with similar molecular structures, including PFBDT-IDTIC, ${ }^{24}$ PF2-DTSi, ${ }^{25}$ PN1, ${ }^{26}$ PF3-DTCO, ${ }^{27}$ and PSF-IDIC, ${ }^{28}$ were developed by modifying either the fusedring SMA building blocks or donor units and achieved PCEs of over $10 \%$ in their all-PSCs. Moreover, by incorporating the booming fused-ring SMA building blocks of Y5 derivatives with different alkyl chain lengths, few polymer acceptors with an ultralow band gap of $\sim 1.40 \mathrm{eV}$, named A701, ${ }^{29}$ PTPBT-

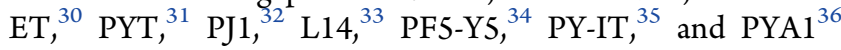
have been produced and have demonstrated high PCEs of 11$15 \%$ in the resulting all-PSCs. In addition to high PCE, good long-term thermal stability is also a key factor in the practical application of all-PSCs. Very recently, a new class of acceptor, that is, a nonconjugated polymer named PF1-TS4 based on the IDIC16 building block linked by a thioalkyl segment in the mainchain was first developed by our group and showed excellent photophysical properties with a high absorption coefficient, low optical band gap, and high lowest unoccupied molecular orbital (LUMO) level, ${ }^{37}$ which are similar to the IDIC16-based full-conjugated polymer acceptors. ${ }^{23-25}$ Moreover, compared to IDIC16 with a strong tendency of selfaggregation in blends due to its highly ordered multicrystallinity, $^{23,25,37}$ the PF1-TS4 film presents reduced crystallinity and good compatibility with the polymer donor in active layers, thus leading to an improved morphological stability in blend films. As a result, its all-PSCs achieved excellent long-term thermal stability under annealing at $85^{\circ} \mathrm{C}$. Although nonconjugated PF1-TS4 has great potential for practical applications in all-PSCs, it still shows a relatively low device efficiency compared to those fused-ring SMA-based fullconjugated polymer acceptor counterparts. ${ }^{23-25}$

The strategy to synthesize terpolymer acceptors by introducing two different electron-withdrawing (A1 and $\mathrm{A} 2$ ) or electron-donating (D1 and D2) moieties forming a $1 \mathrm{D} / 2 \mathrm{~A}$ or $2 \mathrm{D} / 1 \mathrm{~A}$ structure in the molecular backbones has been considered as an efficient approach to synergistically optimize the molecular absorption, energy levels, electron mobility, and aggregation of the resulting polymers. ${ }^{30,38-46}$ However, the application of terpolymer acceptors based on two different fused-ring SMA building blocks in all-PSCs has not been reported. Herein, to explore the effect of the terpolymer strategy for nonconjugated polymer acceptors, we developed a series of nonconjugated terpolymer acceptors with a $1 \mathrm{D} / 2 \mathrm{~A}$ structure by incorporating two different fused-ring SMA building blocks of five-ring-fused IDIC $16^{23,25}$ with long hexadecyl sidechains and seven-ring-fused ITIC $^{1}$ with short 4-hexylphenyl sidechains. The different molecular structures of IDIC16 and ITIC resulted in significantly different solubility, absorption, crystallinity, and aggregation properties. ${ }^{1,23,47,48}$ By simply modulating the IDIC16/ITIC ratios, four nonconjugated terpolymer acceptors (PF1-TS4- $x x$, where $x x$ is the molar percentage of ITIC unit relative to the total SMA building blocks) were synthesized, and the corresponding optical and electrical properties are conveniently tailored. Matched with a wide band gap polymer donor PM6, ${ }^{49}$ allPSCs from PF1-TS4-60 achieved a PCE of 9.31\% with a high open-circuit voltage $\left(V_{\mathrm{oc}}\right)$ of $0.99 \mathrm{~V}, J_{\mathrm{sc}}$ of $15.30 \mathrm{~mA} \mathrm{~cm}^{-2}$, and fill factor (FF) of $61.4 \%$, which is $\sim 50 \%$ higher PCE than the copolymer PF1-TS4 based one (6.17\%).

\section{RESULTS AND DISCUSSION}

The molecular structures of five nonconjugated polymer acceptors, including the copolymer PF1-TS4 and the terpolymers PF1-TS4- $x x(x x=20,40,60$, and 80), are shown in Scheme 1. The copolymer PF1-TS4-100, with a $100 \%$ molar percentage of ITIC unit, possessed a very poor solubility to be used for device fabrication. As shown in Scheme S1, these polymer acceptors were systematically synthesized by Stille-coupling polymerization of three monomers, including two brominated fused-ring SMA building blocks of IDIC16-Br and ITIC-Br with different feeding ratios and a stannylated nonconjugated linkage of bis(trimethylstannyl)-substituted dithiobutyl bithiophenes (TS4- 
(a)

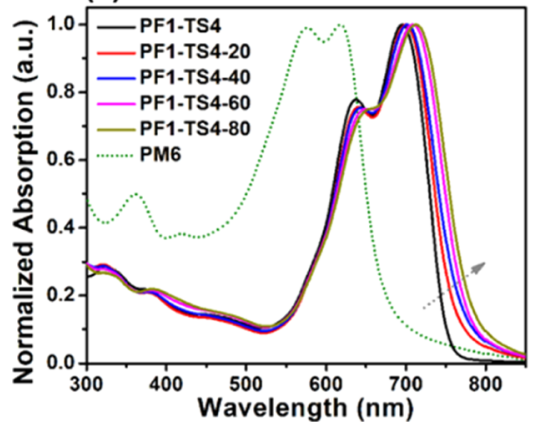

(b)

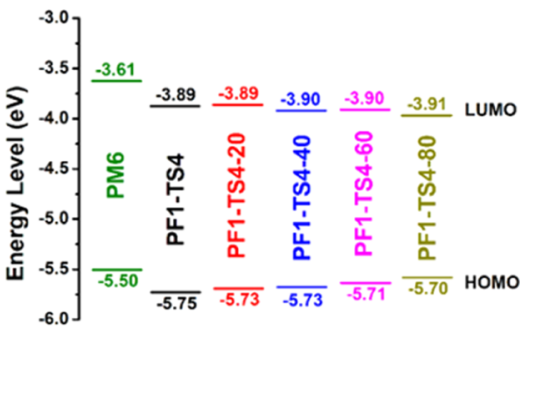

Figure 1. (a) Normalized absorption spectra in neat films and (b) energy-level diagrams of active layer photovoltaic materials in neat films.

(a)

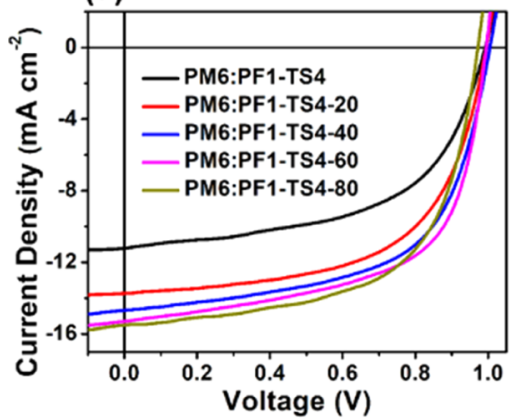

(c)

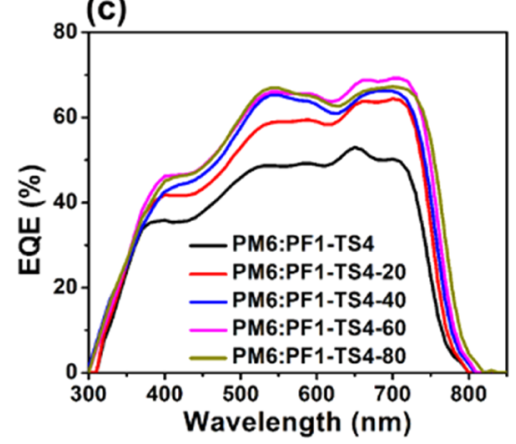

(b)

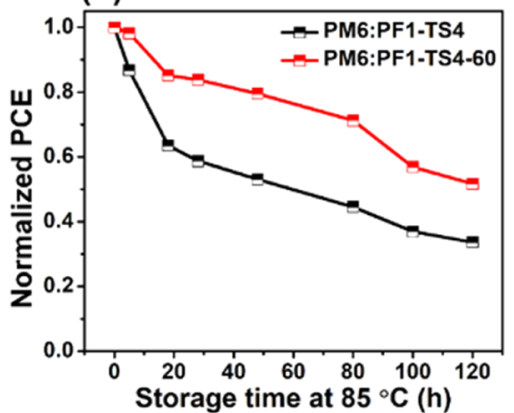

(d)

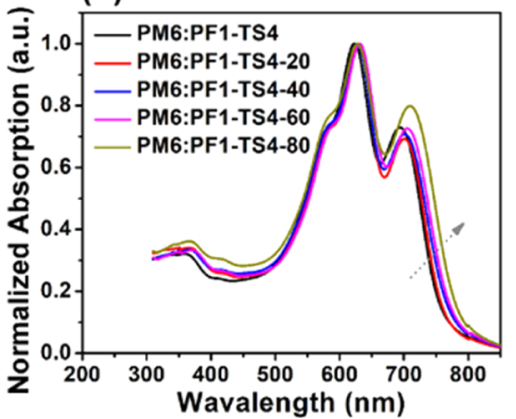

Figure 2. (a) $J-V$ plots of the all-PSCs under the illumination of $A M 1.5 \mathrm{G}, 100 \mathrm{~mW} \mathrm{~cm}^{-2}$. (b) Thermal stability of the devices with an annealing temperature of $85^{\circ} \mathrm{C}$ in the $\mathrm{N}_{2}$-filled glovebox under dark conditions. (c) External quantum efficiency (EQE) spectra of all-PSCs. (d) Normalized absorption spectra of all-polymer active layers based on PM6 and different nonconjugated polymer acceptors.

$\mathrm{Sn})$. Because of the long alkyl sidechains in the IDIC16 building block and the nonconjugated flexible TS4 segment, terpolymers (except PF1-TS4-100) show good solubility in common warm organic solvents.

As shown in Figure 1a of the ultraviolet-visible (UV-vis) absorption spectra, the effect of the third ITIC unit on molecular optical properties of the nonconjugated polymer acceptors in neat films was investigated. With the increase of ITIC content, these polymer acceptors display gradually redshifted absorption spectra, which follow well the trend of broadened absorption spectra from IDIC16 to ITIC (Figure S1). Among them, the absorption onset of PF1-TS4-80 with the smallest band gap $(\sim 1.55 \mathrm{eV})$ is located at $\sim 800 \mathrm{~nm}$, which is red-shifted by $\sim 50 \mathrm{~nm}$ compared to the original PF1TS4. Notably, compared to its fused-ring SMA building block ITIC, PF1-TS4-60 and PF1-TS4-80 show identical absorption onsets but red-shifted and sharper absorption peaks (Figure S1), indicating the improved optical absorption capability. As shown in Figure S2 of cyclic voltammograms and Figure 1b of the corresponding energy-level diagrams, although our random ternary polymerization strategy obviously broadens the molecular absorption spectra, the molecular energy levels of these polymer acceptors only show slight variations in their LUMO levels $(\sim 0.02 \mathrm{eV})$, which may be due to the similar LUMO levels of the two building blocks IDIC16 $6^{37}(-3.87 \mathrm{eV})$ and $\operatorname{ITIC}^{50,51}(-3.85 \mathrm{eV})$. On the other hand, their HOMO levels are varied $(\sim 0.05 \mathrm{eV})$ to a larger extent compared to the LUMO levels when the ITIC portion in terpolymers is increased, which is consistent with their gradually decreased optical band gap and conducive to achieving a better tradeoff between $V_{\text {oc }}$ and $J_{\text {sc }}$ in all-PSC devices. As shown in Figure S3a, with the increase of ITIC content, the electron mobility $\left(\mu_{\mathrm{e}}\right)$ of polymer acceptors increases first and then decreases, as measured by means of a space charge limited current (SCLC) method, which may be attributed to the improved intermolecular interactions; however in the case of PF1-TS480 , the relatively poor solubility in a nonhalogenated solvent (o-xylene) leads to a nonoptimized morphology and therefore slightly lower electron mobility. Among them, the terpolymer 
PF1-TS4-60 has the highest $\mu_{\mathrm{e}}$ value of $3.54 \times 10^{-5} \mathrm{~cm}^{2} \mathrm{~V}^{-1}$ $\mathrm{s}^{-1}$, which is $\sim 2.5$ times that of the copolymer PF1-TS4.

To investigate the effect on photovoltaic performance of the introduction of the third ITIC unit into the nonconjugated PF1-TS4 backbone, all-PSCs with a device structure of ITO/ PEDOT:PSS/active layer/PFN-Br/Ag were fabricated. As opposed to the PM6-based all-PSCs with the nonconjugated copolymer acceptor PF1-TS4 in our previous work, ${ }^{33}$ the PM6-based active layers with different nonconjugated polymer acceptors (copolymer PF1-TS4 and terpolymers PF1-TS4- $x x$ ) in this work were prepared by spin-coating the blend solutions with a D/A ratio of $1: 1$ (wt/wt \%) and a total solid concentration of $16 \mathrm{mg} \mathrm{mL}^{-1}$ that was prepared using the nonhalogenated solvent $o$-xylene and the high boiling point additive of 1-chloronaphthalene ( $2 \%$ in v/v). Detailed fabrication processes of all-PSCs are described in Supporting Information, and the corresponding current density-voltage $(J-V)$ curves and photovoltaic parameters are summarized in Figure $2 \mathrm{a}$ and Table 1, respectively. With the increase of ITIC

Table 1. Photovoltaic Data of the PM6-Based All-PSCs with Different Nonconjugated Polymer Acceptors

\begin{tabular}{|c|c|c|c|c|}
\hline $\mathrm{D}: \mathrm{A}$ & $\begin{array}{l}V_{\mathrm{oc}} \\
{[\mathrm{V}]}\end{array}$ & {$\left[\mathrm{mA} \mathrm{cm}^{J_{\mathrm{sc}}}\right]^{a}$} & $\begin{array}{l}\mathrm{FF} \\
{[\%]}\end{array}$ & $\operatorname{PCE}[\%]^{b}$ \\
\hline PM6:PF1-TS4 & 0.99 & $11.21(10.91)$ & 55.6 & $6.17(5.80 \pm 0.23)$ \\
\hline PM6:PF1-TS4-20 & 0.99 & $13.73(13.31)$ & 59.7 & $8.12(7.73 \pm 0.27)$ \\
\hline PM6:PF1-TS4-40 & 0.99 & $14.67(14.32)$ & 60.8 & $8.83(8.56 \pm 0.19)$ \\
\hline PM6:PF1-TS4-60 & 0.99 & $15.30(14.94)$ & 61.4 & $9.31(9.13 \pm 0.16)$ \\
\hline PM6:PF1-TS4-80 & 0.97 & $15.51(15.03)$ & 60.3 & $9.16(8.87 \pm 0.20)$ \\
\hline
\end{tabular}

contents, these polymer acceptors achieved gradually improved $J_{\mathrm{sc}}$ from 11.21 to $15.51 \mathrm{~mA} \mathrm{~cm}{ }^{-2}$ and almost unchanged $V_{\mathrm{oc}}$ of 0.97-0.99 V in all-PSCs, which means that the random ternary polymerization strategy by coupling two fused-ring SMA building blocks (IDIC16 and ITIC) and a nonconjugated donor linkage (TS4) can effectively balance the tradeoff between $J_{\text {sc }}$ and $V_{\text {oc }}$ in all-PSCs. Moreover, all terpolymers demonstrated the obviously increased FF of 59.7-61.4\% in allPSCs compared to the copolymer PF1-TS4, while PF1-TS4-60 obtained the highest FF of $61.4 \%$. As a result, the PM6:PF1TS4-60-based all-PSCs achieved a champion PCE of 9.31\%, which is $\sim 50 \%$ higher than that of PM6:PF1-TS4-based ones (6.17\%, note: the different batches of PF1-TS4 and different device architectures used in this work may cause the different device performances from our previous study ${ }^{37}$ ). The highest PCE of PF1-TS4-60 among all-PSCs indicates the strong benefit of our random ternary polymerization strategy with the finely regulated IDIC16/ITIC ratio. The influence of our terpolymer strategy on the thermal stability of devices was also investigated. As shown in Figure 2b, the PM6:PF1-TS4-60based all-PSC has a better thermal stability in comparison with the PM6:PF1-TS4-based all-PSC at an annealing temperature of $85{ }^{\circ} \mathrm{C}$ in the $\mathrm{N}_{2}$-filled glovebox under dark conditions. Because these terpolymer acceptors are formed in a random order of TS4, ITIC and IDIC16 random distribution in backbones may cause batch-to-batch variations in performance. Therefore, two additional batches of terpolymer PF1-TS4-60 were synthesized by using the same procedure as the first batch to investigate the effect of batch-to-batch variations on the device performance. As shown in Figure S4 and Table S2, three batches of PF1-TS4-60 achieved PCEs varying from 8.54 to $9.31 \%$, even though $V_{\mathrm{oc}}$ and $J_{\mathrm{sc}}$ pronounced good reproducibility, which suggests that PF1-TS4-60 has a slight batch-to-batch dependence on device performance. Moreover, as shown in Scheme S2 and Table S3, among the all-PSCs based on IDIC16-containing polymer acceptors, the all-PSCs based on PF1-TS4-60 in this work achieved a PCE in the high end.

As shown in Figure 2c, with the increase of ITIC content in nonconjugated polymer acceptors, the corresponding all-PSCs display gradually red-shifted EQE responses, which are consistent with the absorption spectra of polymer acceptors in neat films (Figure 1a) and their related all-polymer blend films (Figure 2d). Compared to the PF1-TS4-based all-PSCs, all the all-PSCs based on terpolymers exhibit dramatically increased EQE values over the whole wavelength region of 400-800 nm, while the PF1-TS4-60-based device obtained the highest EQE value close to $70 \%$ and the PF1-TS4-80-based device presents the broadest EQE response, indicating that our terpolymer strategy by introducing ITIC as the third unit can effectively improve the conversion of incident photons to electrons. The integrated $J_{s c}$ values calculated from the corresponding EQE curves are 10.91, 13.31, 14.32, 14.94, and $15.03 \mathrm{~mA} \mathrm{~cm}^{-2}$ for the devices based on PM6:PF1-TS4, PM6:PF1-TS4-20, PM6:PF1-TS4-40, PM6:PF1-TS4-60, and PM6:PF1-TS4-80, respectively, which are all well consistent with the measured $J_{\mathrm{sc}}$ values from the corresponding $J-V$ plots, with deviations of less than $5 \%$.

Photoluminescence (PL) quenching experiments were performed to study the exciton dissociation and charge transfer behavior of all-polymer blend films. As shown in the PL spectra of nonconjugated polymer acceptor neat films in Figure 3a, the terpolymers PF1-TS4- $x x$ show red-shifted PL peaks and onsets, compared to the copolymer PF1-TS4, as well as significantly increased PL intensities. Moreover, terpolymers PF1-TS4-60 and PF1-TS4-80 with an IDIC16/ITIC molar ratio $<1$ produce higher $\mathrm{PL}$ intensities compared to those terpolymers of PF1-TS4-20 and PF1-TS4-40 with an IDIC16/ ITIC molar ratio $>1$, while the PL intensities are found to be quite similar between the former two and the latter two, which may be due to the better coplanarity and increased intermolecular interaction of the ITIC unit. Because the nonradiative recombination of devices is generally inversely proportional to the PL yield of the pristine film of photovoltaic materials, ${ }^{52}$ the higher PL efficiencies of the terpolymers PF1TS4-60 and PF1-TS4-80 are in favor of a lower nonradiative recombination loss in the resulting all-PSCs. The abovementioned results may partially explain why terpolymers PF1TS4-60 and PF1-TS4-80 with obviously red-shifted absorption spectra exhibit little change in their LUMO levels and $V_{\mathrm{oc}}$ values in all-PSCs compared to the original PF1-TS4. As shown in Figure $3 b-h$, compared to the polymer acceptor neat films, the corresponding PM6:terpolymer blend films display significantly increased PL quenching efficiencies of $80-90 \%$ in comparison with the PM6:PF1-TS4 blend film (64.3\%). The PM6:PF1-TS4-60 blend film has the highest PL quenching efficiency of $88.2 \%$. A similar phenomenon is also found in the PL measurements of the PM6 neat film and related blend films (Figure $3 \mathrm{~g}, \mathrm{~h}$ ), indicating better compatibility and more efficient photoinduced hole and electron transfer between PM6 and terpolymers in devices, especially between PM6 and PF1-TS4-60, which is consistent with the trend of $J_{\mathrm{sc}}$ in their 

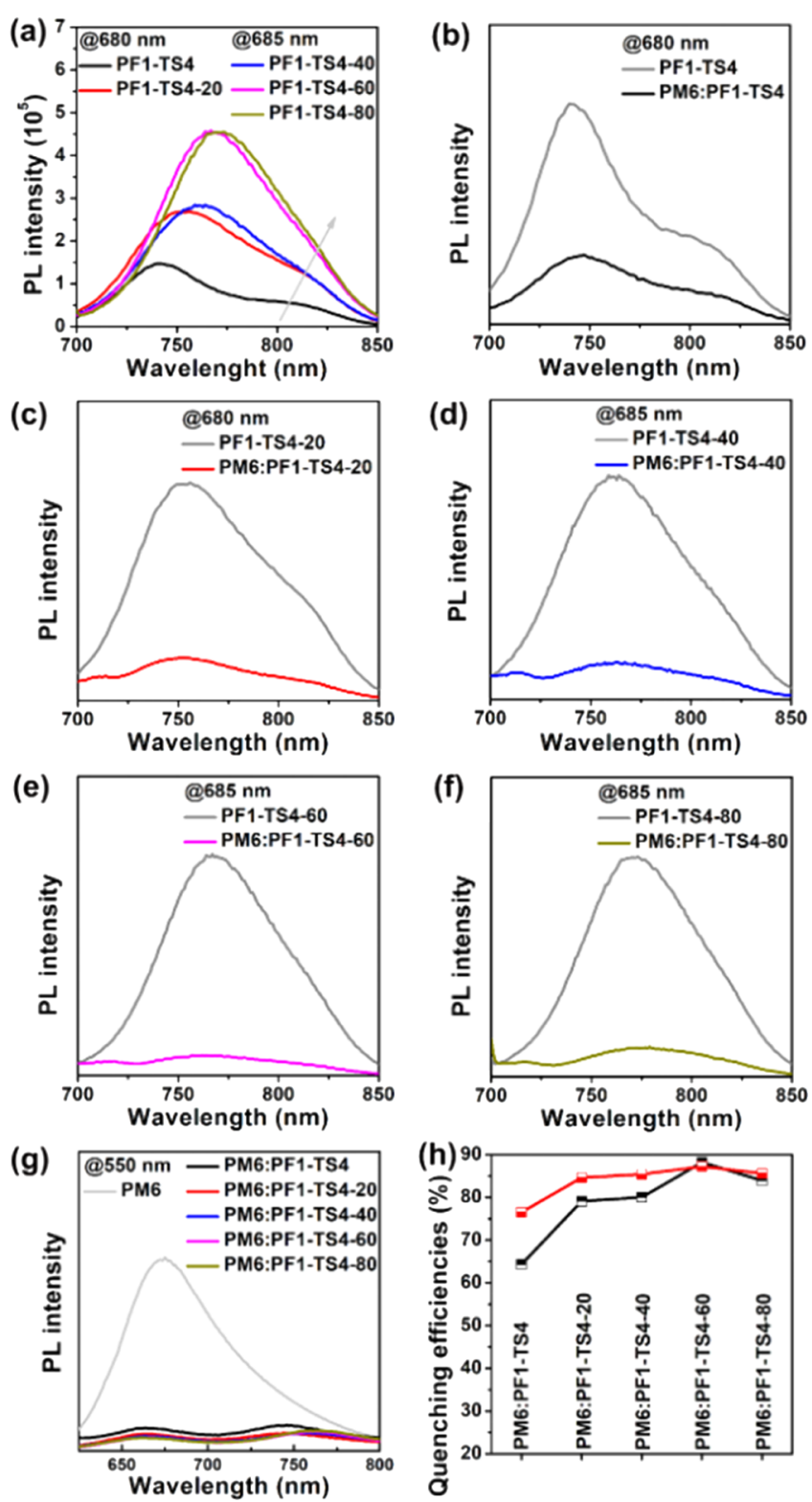

Figure 3. PL spectra of these polymer photovoltaic materials in neat films and related blend films (a) excited at 680 or $685 \mathrm{~nm}$ for polymer acceptors; $(b, c)$ excited at $680 \mathrm{~nm}$ for PF1-TS4, PF1-TS4-20, and related blend films; (d-f) excited at $\sim 685 \mathrm{~nm}$ for PF1-TS4-40, PF1TS4-60, PF1-TS4-80, and related blend films; and (g) excited at $~ 550$ $\mathrm{nm}$ for PM6 and related blend films. (h) PL quenching efficiencies of blend films relative to the related polymer acceptor neat films (black line, extracted from $b$ to f) and the PM6 neat film (red line, extracted from $\mathrm{g}$ ).

all-PSCs. Compared to the PM6:PF1-TS4-60 film, the PM6:PF1-TS4-80 film shows slightly lower PL quenching efficiencies, which is probably due to the relatively weak compatibility between PM6 and PF1-TS4-80 in blend films and the poor solubility of PF1-TS4-80, which is consistent with the fact that the PM6:PF1-TS4-80 film has an obviously stronger shoulder peak belonging to polymer acceptors (see Figure 2d).

The atomic force microscopy (AFM) and transmission electron microscopy (TEM) measurements were carried out to probe the effect of the introduction of the third ITIC unit into the molecular backbone of nonconjugated polymer acceptors on the nanoscale morphologies of polymer neat films and their related all-polymer active layers. As shown in Figure $4 a-e$ of AFM images, with the increase of ITIC content, the neat films of these nonconjugated polymer acceptors show gradually increased root-mean-square (RMS) roughness values from 0.27 to $1.03 \mathrm{~nm}$, which may be due to the increased intermolecular interaction and decreased solubility resulting from the relatively larger backbone of ITIC. As shown in Figure 4f, the PM6:PF1-TS4 blend film displays a very coarse domain structure, resulting in a very rough surface with an excessive RMS roughness of $4.07 \mathrm{~nm}$. With the progressively increasing ITIC content in the polymer acceptors, it is clearly seen that the related all-polymer blend active layers produce a gradually dwindling domain structure and reducing RMS roughness values from 4.07 to $1.63 \mathrm{~nm}$ in turn (Figures $4 \mathrm{~g}-\mathrm{j}$ ), which is contrary to the trend of the RMS roughness values of the AFM images of polymer acceptor neat films. This opposite trend indicates that the introduction of the third ITIC building block into the polymer acceptor backbone can improve the compatibility of the terpolymer with the polymer donor PM6. Among them, the PM6:PF1-TS4-60 and PM6:PF1-TS4-80 blend films show more suitable domain structures with a smoother surface and a smaller RMS roughness of $\sim 1.65 \mathrm{~nm}$. Moreover, this trend is confirmed by the TEM images, as shown in Figures 4k-o, where the PM6:PF1-TS4-60 blend film demonstrates a more uniform fibril structure with an appropriate domain size, which can facilitate the charge transport of its all-PSCs. As shown in Figure S3b and Table $S 1$, with the increasing ITIC content in the polymer acceptors, the related all-polymer blend films also show gradually improved hole mobilities from $0.84 \times 10^{-4} \mathrm{~cm}^{2} \mathrm{~V}^{-1} \mathrm{~s}^{-1}$ for PM6:PF1-TS4 to $1.94 \times 10^{-4} \mathrm{~cm}^{2} \mathrm{~V}^{-1} \mathrm{~s}^{-1}$ for PM6:PF1-TS480 , while their electron mobilities firstly increase from $1.44 \times$ $10^{-5} \mathrm{~cm}^{2} \mathrm{~V}^{-1} \mathrm{~s}^{-1}$ for PM6:PF1-TS4 to $2.94 \times 10^{-5} \mathrm{~cm}^{2} \mathrm{~V}^{-1} \mathrm{~s}^{-1}$ for PM6:PF1-TS4-60 and then decrease to $2.54 \times 10^{-5} \mathrm{~cm}^{2}$ $\mathrm{V}^{-1} \mathrm{~s}^{-1}$ for PM6:PF1-TS4-80. As a result, the PM6:PF1-TS460 blend films exhibit more balanced hole and electron mobilities, which implies less accumulation of space charge in devices.

\section{CONCLUSIONS}

In conclusion, we developed a series of low band gap nonconjugated terpolymer acceptors based on two different fused-ring SMA building blocks IDIC16 and ITIC, where ITIC with a larger $\pi$-fused structure, shorter solubilizing sidechains, and red-shifted absorption spectrum is incorporated as the third component into the copolymer acceptor PF1-TS4 based on the IDIC16 linked by flexible thioalkyl chain-thiophene segments. With the increasing ITIC contents, terpolymers show adjustable photoelectric properties, while the active layer based on the terpolymer PF1-TS4-60 with the 60\% ITIC unit shows an optimized blend morphology, increased PL quenching efficiency, and improved charge transport properties compared to those based on other polymer acceptors. In allPSCs, the one based on PF1-TS4-60 achieved the same $V_{\text {oc }}$ of $0.99 \mathrm{~V}$, but obviously increased $J_{\mathrm{sc}}$ of $15.30 \mathrm{~mA} \mathrm{~cm}^{-2}$ and $\mathrm{FF}$ of $61.4 \%$ compared to the one based on PF1-TS4 $\left(V_{\mathrm{oc}}=0.99 \mathrm{~V}\right.$, $J_{\mathrm{sc}}=11.21 \mathrm{~mA} \mathrm{~cm}{ }^{-2}$, and $\left.\mathrm{FF}=55.6 \%\right)$. As a result, a promising PCE of $9.31 \%$ was obtained by the PF1-TS4-60-based devices, which is $\sim 50 \%$ higher than that of the PF1-TS4-based one $(6.17 \%)$. The results strongly suggest that the ternary polymerization by incorporating two different fused-ring SMA building blocks is a useful approach for the development 

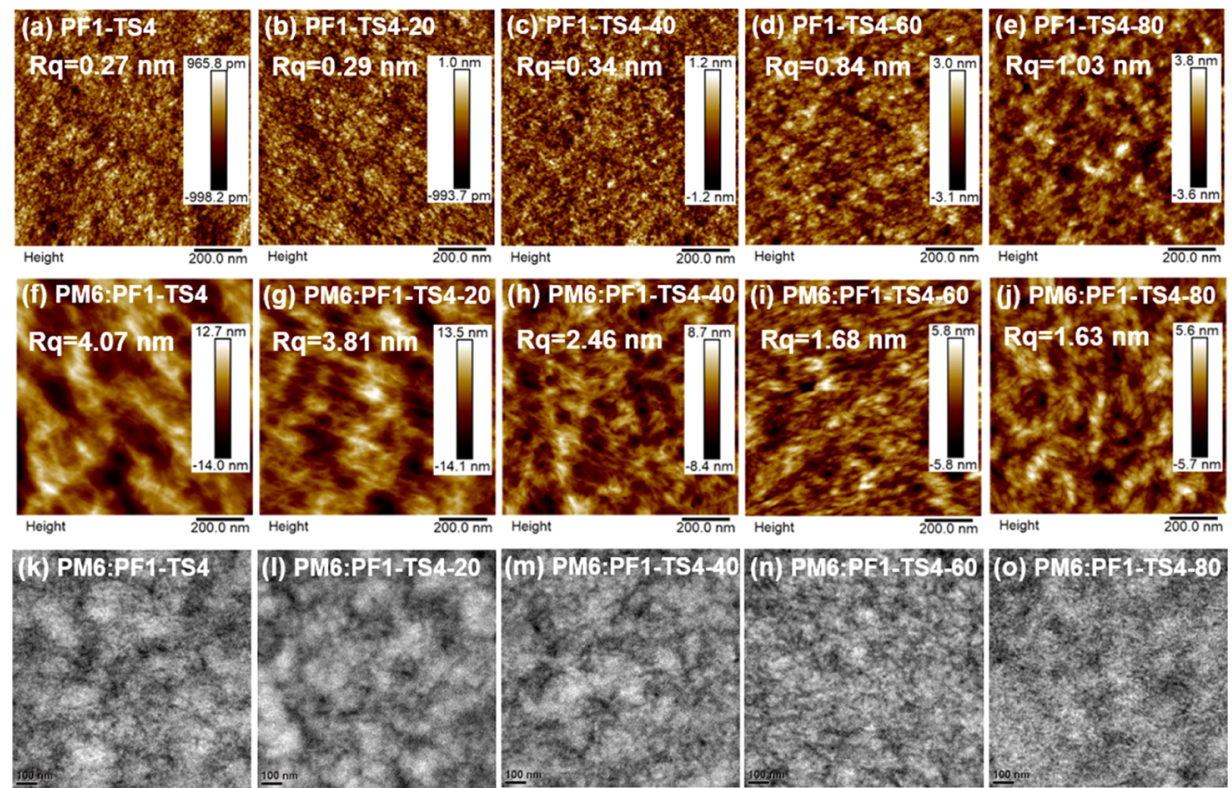

Figure 4. AFM height images of $(a-e)$ nonconjugated polymer acceptor pure films and $(f-j)$ related all-polymer blend films. (k-o) TEM images of the all-polymer blend films.

of high-performance nonconjugated polymer acceptors toward efficient all-PSCs.

\section{EXPERIMENTAL SECTION}

4.1. Materials. A polymer donor PM6, two brominated fused-ring SMA building blocks of IDIC16-Br and ITIC-Br, a monomer TS4-Sn, and a polymer acceptor PF1-TS4 were synthesized according to previous works. The detailed processes of the synthesis of four nonconjugated terpolymer acceptors (PF1-TS4-20, PF1-TS4-40, PF1TS4-60, and PF1-TS4-80) and related device fabrication and characterization are summarized in the Supporting Information.

\section{ASSOCIATED CONTENT}

\section{s) Supporting Information}

The Supporting Information is available free of charge at https://pubs.acs.org/doi/10.1021/acsami.0c17722.

Synthesis of nonconjugated terpolymer acceptors, SCLC mobility measurements, thin-film fabrication and characterization, UV-vis spectra, cyclic voltammograms, device performance of all-PSCs based on PF1-TS4-60 with different batches, and the summary of IDIC16based polymer acceptors and related photovoltaic performance (PDF)

\section{AUTHOR INFORMATION}

\section{Corresponding Authors}

Qunping Fan - Department of Chemistry and Chemical Engineering, Chalmers University of Technology, SE-412 96 Göteborg, Sweden; 다이.org/0000-0002-7268-8065; Email: qunpifan@cityu.edu.hk

Lintao Hou - Guangdong Provincial Key Laboratory of Optical Fiber Sensing and Communications, Guangzhou Key Laboratory of Vacuum Coating Technologies and New Energy Materials, Siyuan Laboratory, Department of Physics, Jinan University, 510632 Guangzhou, China; Email: thlt@ jnu.edu.cn

Ellen Moons - Department of Engineering and Physics, Karlstad University, SE-651 88 Karlstad, Sweden; 다이.org/0000-0002-1609-8909; Email: ellen.moons@ kau.se

Ergang Wang - Department of Chemistry and Chemical Engineering, Chalmers University of Technology, SE-412 96 Göteborg, Sweden; School of Materials Science and Engineering, Zhengzhou University, 450001 Zhengzhou, China; orcid.org/0000-0002-4942-3771;

Email: ergang@chalmers.se

\section{Authors}

Wenyan Su - Guangdong Provincial Key Laboratory of Optical Fiber Sensing and Communications, Guangzhou Key Laboratory of Vacuum Coating Technologies and New Energy Materials, Siyuan Laboratory, Department of Physics, Jinan University, 510632 Guangzhou, China; Department of Chemistry and Chemical Engineering, Chalmers University of Technology, SE-412 96 Göteborg, Sweden; Department of Engineering and Physics, Karlstad University, SE-651 88 Karlstad, Sweden

Ishita Jalan - Department of Engineering and Chemical Sciences, Karlstad University, SE-651 88 Karlstad, Sweden

Yufei Wang - Guangdong Provincial Key Laboratory of Optical Fiber Sensing and Communications, Guangzhou Key Laboratory of Vacuum Coating Technologies and New Energy Materials, Siyuan Laboratory, Department of Physics, Jinan University, 510632 Guangzhou, China

Wenhong Peng - Department of Chemistry and Chemical Engineering, Chalmers University of Technology, SE-412 96 Göteborg, Sweden; School of Materials Science and Engineering, Jiangsu Key Laboratory of Environmentally Friendly Polymeric Materials, Jiangsu Engineering Laboratory of Light-Electricity-Heat Energy-Converting Materials and Applications, Changzhou University, 213164 Changzhou, China

Tao Guo - Department of Chemistry and Chemical Engineering, Chalmers University of Technology, SE-412 96 Göteborg, Sweden; School of Chemistry and Chemical Engineering, Henan University of Technology, 450001 Zhengzhou, China 
Weiguo Zhu - School of Materials Science and Engineering, Jiangsu Key Laboratory of Environmentally Friendly Polymeric Materials, Jiangsu Engineering Laboratory of Light-Electricity-Heat Energy-Converting Materials and Applications, Changzhou University, 213164 Changzhou, China; orcid.org/0000-0002-4244-2638

Donghong Yu - Department of Chemistry and Bioscience, Aalborg University, DK-9220 Aalborg, Denmark; SinoDanish Center for Education and Research, DK-8000 Aarhus, Denmark

Complete contact information is available at: https://pubs.acs.org/10.1021/acsami.0c17722

\section{Notes}

The authors declare no competing financial interest.

\section{ACKNOWLEDGMENTS}

We thank the Swedish Research Council (2015-04853, 201606146, and 2019-04683), the Swedish Research Council Formas, and the Knut and Alice Wallenberg Foundation (2017.0186, 2016.0059) for financial support. E.M. thanks the Swedish Energy Council for financial support (project 485981). W.S. thanks the project funded by China Postdoctoral Science Foundation (2020M673054), Postdoctoral Fund of Jinan University, and National Natural Science Foundation of China (22005121). L.H. thanks the NSFC project (61774077) for financial support. The support from Sino-Danish Centre for Education and Research and the Open Fund of the State Key Laboratory of Luminescent Materials and Devices (South China University of Technology, 2020-skllmd-07) is fully acknowledged. Leif K. E. Ericsson is acknowledged for the helpful discussion.

\section{REFERENCES}

(1) Lin, Y.; Wang, J.; Zhang, Z.-G.; Bai, H.; Li, Y.; Zhu, D.; Zhan, X. An Electron Acceptor Challenging Fullerenes for Efficient Polymer Solar Cells. Adv. Mater. 2015, 27, 1170-1174.

(2) Yuan, J.; Zhang, Y.; Zhou, L.; Zhang, G.; Yip, H.-L.; Lau, T.-K.; Lu, X.; Zhu, C.; Peng, H.; Johnson, P. A.; Leclerc, M.; Cao, Y.; Ulanski, J.; Li, Y.; Zou, Y. Single-Junction Organic Solar Cell with over 15\% Efficiency Using Fused-Ring Acceptor with ElectronDeficient Core. Joule 2019, 3, 1140-1151.

(3) Yao, J.; Qiu, B.; Zhang, Z.; Xue, L.; Wang, R.; Zhang, C.; Chen, S.; Zhou, Q.; Sun, C.; Yang, C.; Xiao, M.; Meng, L.; Li, Y. Cathode engineering with perylene-diimide interlayer enabling over $17 \%$ efficiency single-junction organic solar cells. Nat. Commun. 2020, $11,2726$.

(4) Liu, Q.; Jiang, Y.; Jin, K.; Qin, J.; Xu, J.; Li, W.; Xiong, J.; Liu, J.; Xiao, Z.; Sun, K.; Yang, S.; Zhang, X.; Ding, L. 18\% Efficiency organic solar cells. Sci. Bull. 2020, 65, 272-275.

(5) Meng, L.; Zhang, Y.; Wan, X.; Li, C.; Zhang, X.; Wang, Y.; Ke, X.; Xiao, Z.; Ding, L.; Xia, R.; Yip, H. L.; Cao, Y.; Chen, Y. Organic and solution-processed tandem solar cells with $17.3 \%$ efficiency. Science 2018, 361, 1094-1098.

(6) Lin, Y.; Adilbekova, B.; Firdaus, Y.; Yengel, E.; Faber, H.; Sajjad, M.; Zheng, X.; Yarali, E.; Seitkhan, A.; Bakr, O. M.; El-Labban, A.; Schwingenschlogl, U.; Tung, V.; McCulloch, I.; Laquai, F.; Anthopoulos, T. D. 17\% Efficient Organic Solar Cells Based on Liquid Exfoliated WS2 as a Replacement for PEDOT:PSS. Adv. Mater. 2019, 31, 1902965.

(7) Liu, L.; Kan, Y.; Gao, K.; Wang, J.; Zhao, M.; Chen, H.; Zhao, C.; Jiu, T.; Jen, A.-K.-Y.; Li, Y. Graphdiyne Derivative as Multifunctional Solid Additive in Binary Organic Solar Cells with 17.3\% Efficiency and High Reproductivity. Adv. Mater. 2020, 32, 1907604.
(8) Cui, Y.; Yao, H.; Zhang, J.; Xian, K.; Zhang, T.; Hong, L.; Wang, Y.; Xu, Y.; Ma, K.; An, C.; He, C.; Wei, Z.; Gao, F.; Hou, J. SingleJunction Organic Photovoltaic Cells with Approaching 18\% Efficiency. Adv. Mater. 2020, 32, 1908205.

(9) Wang, T.; Sun, R.; Shi, M.; Pan, F.; Hu, Z.; Huang, F.; Li, Y.; Min, J. Solution-Processed Polymer Solar Cells with over 17\% Efficiency Enabled by an Iridium Complexation Approach. Adv. Energy Mater. 2020, 10, 2000590.

(10) Liu, T.; Ma, R.; Luo, Z.; Guo, Y.; Zhang, G.; Xiao, Y.; Yang, T.; Chen, Y.; Li, G.; Yi, Y.; Lu, X.; Yan, H.; Tang, B. Concurrent improvement in $\mathrm{J}_{\mathrm{sc}}$ and $\mathrm{V}_{\mathrm{oc}}$ in high-efficiency ternary organic solar cells enabled by a red-absorbing small-molecule acceptor with a high LUMO level. Energy Environ. Sci. 2020, 13, 2115-2123.

(11) Genene, Z.; Mammo, W.; Wang, E.; Andersson, M. R. Recent Advances in n-Type Polymers for All-Polymer Solar Cells. Adv. Mater. 2019, 31, 1807275.

(12) Wang, G.; Melkonyan, F. S.; Facchetti, A.; Marks, T. J. AllPolymer Solar Cells: Recent Progress, Challenges, and Prospects. Angew. Chem., Int. Ed. 2019, 58, 4129-4142.

(13) Yang, J.; Xiao, B.; Tang, A.; Li, J.; Wang, X.; Zhou, E. AromaticDiimide-Based n-Type Conjugated Polymers for All-Polymer Solar Cell Applications. Adv. Mater. 2019, 31, 1804699.

(14) Lee, C.; Lee, S.; Kim, G.-U.; Lee, W.; Kim, B. J. Recent Advances, Design Guidelines, and Prospects of All-Polymer Solar Cells. Chem. Rev. 2019, 119, 8028-8086.

(15) (a) Chen, H.; Guo, Y.; Chao, P.; Liu, L.; Chen, W.; Zhao, D.; $\mathrm{He}, \mathrm{F}$. A chlorinated polymer promoted analogue co-donors for efficient ternary all-polymer solar cells. Sci. China Chem. 2019, 62, 238-244. (b) Guo, Y.; Li, Y.; Awartani, O.; Han, H.; Zhao, J.; Ade, H.; Yan, H.; Zhao, D. Improved Performance of All-Polymer Solar Cells Enabled by Naphthodiperylenetetraimide-Based Polymer Acceptor. Adv. Mater. 2017, 29, 1700309.

(16) Yan, H.; Chen, Z.; Zheng, Y.; Newman, C.; Quinn, J. R.; Dötz, F.; Kastler, M.; Facchetti, A. A high-mobility electron-transporting polymer for printed transistors. Nature 2009, 457, 679-686.

(17) Fan, B.; Zhong, W.; Ying, L.; Zhang, D.; Li, M.; Lin, Y.; Xia, R.; Liu, F.; Yip, H. L.; Li, N.; Ma, Y.; Brabec, C. J.; Huang, F.; Cao, Y. Surpassing the $10 \%$ efficiency milestone for $1-\mathrm{cm}^{2}$ all-polymer solar cells. Nat. Commun. 2019, 10, 4100.

(18) Zhu, L.; Zhong, W.; Qiu, C.; Lyu, B.; Zhou, Z.; Zhang, M.; Song, J.; Xu, J.; Wang, J.; Ali, J.; Feng, W.; Shi, Z.; Gu, X.; Ying, L.; Zhang, Y.; Liu, F. Aggregation-Induced Multilength Scaled Morphology Enabling $11.76 \%$ Efficiency in All-Polymer Solar Cells Using Printing Fabrication. Adv. Mater. 2019, 31, 1902899.

(19) Xu, Y.; Yuan, J.; Liang, S.; Chen, J.-D.; Xia, Y.; Larson, B. W.; Wang, Y.; Su, G. M.; Zhang, Y.; Cui, C.; Wang, M.; Zhao, H.; Ma, W. Simultaneously Improved Efficiency and Stability in All-Polymer Solar Cells by a P-i-N Architecture. ACS Energy Lett. 2019, 4, 2277-2286.

(20) Sun, H.; Tang, Y.; Koh, C. W.; Ling, S.; Wang, R.; Yang, K.; Yu, J.; Shi, Y.; Wang, Y.; Woo, H. Y.; Guo, X. High-Performance AllPolymer Solar Cells Enabled by an n-Type Polymer Based on a Fluorinated Imide-Functionalized Arene. Adv. Mater. 2019, 31, 1807220.

(21) Zhao, R.; Wang, N.; Yu, Y.; Liu, J. Organoboron Polymer for 10\% Efficiency All-Polymer Solar Cells. Chem. Mater. 2020, 32, $1308-1314$.

(22) Li, Y.; Meng, H.; Liu, T.; Xiao, Y.; Tang, Z.; Pang, B.; Li, Y.; Xiang, Y.; Zhang, G.; Lu, X.; Yu, G.; Yan, H.; Zhan, C.; Huang, J.; Yao, J. 8.78\% Efficient All-Polymer Solar Cells Enabled by Polymer Acceptors Based on a $\mathrm{B} \leftarrow \mathrm{N}$ Embedded Electron-Deficient Unit. Adv. Mater. 2019, 31, 1904585.

(23) Zhang, Z.-G.; Yang, Y.; Yao, J.; Xue, L.; Chen, S.; Li, X.; Morrison, W.; Yang, C.; Li, Y. Constructing a Strongly Absorbing Low-Bandgap Polymer Acceptor for High-Performance All-Polymer Solar Cells. Angew. Chem., Int. Ed. 2017, 56, 13503-13507.

(24) Yao, H.; Bai, F.; Hu, H.; Arunagiri, L.; Zhang, J.; Chen, Y.; Yu, H.; Chen, S.; Liu, T.; Lai, J. Y. L.; Zou, Y.; Ade, H.; Yan, H. Efficient All-Polymer Solar Cells based on a New Polymer Acceptor Achieving 
10.3\% Power Conversion Efficiency. ACS Energy Lett. 2019, 4, 417422.

(25) Fan, Q.; Su, W.; Chen, S.; Kim, W.; Chen, X.; Lee, B.; Liu, T.; Méndez-Romero, U. A.; Ma, R.; Yang, T.; Zhuang, W.; Li, Y.; Li, Y.; Kim, T.-S.; Hou, L.; Yang, C.; Yan, H.; Yu, D.; Wang, E. Mechanically Robust All-Polymer Solar Cells from Narrow Band Gap Acceptors with Hetero-Bridging Atoms. Joule 2020, 4, 658-672.

(26) Wu, J.; Meng, Y.; Guo, X.; Zhu, L.; Liu, F.; Zhang, M. Allpolymer solar cells based on a novel narrow bandgap polymer acceptor with power conversion efficiency over $10 \%$. J. Mater. Chem. A 2019, 7, 16190-16196.

(27) Fan, Q.; Ma, R.; Liu, T.; Su, W.; Peng, W.; Zhang, M.; Wang, Z.; Wen, X.; Cong, Z.; Luo, Z.; Hou, L.; Liu, F.; Zhu, W.; Yu, D.; Yan, H.; Wang, E. 10.13\% Efficiency All-Polymer Solar Cells Enabled by Improving the Optical Absorption of Polymer Acceptors. Sol. RRL 2020, 4, 2000142.

(28) Huang, S.; Wu, F.; Liu, Z.; Cui, Y.; Chen, L.; Chen, Y. Novel polymer acceptors achieving $10.18 \%$ efficiency for all-polymer solar cells. J. Energy Chem. 2021, 53, 63-68.

(29) Tang, A.; Li, J.; Zhang, B.; Peng, J.; Zhou, E. Low-Bandgap nType Polymer Based on a Fused-DAD-Type Heptacyclic Ring for AllPolymer Solar Cell Application with a Power Conversion Efficiency of 10.7\%. ACS Macro Lett. 2020, 9, 706-712.

(30) Du, J.; Hu, K.; Meng, L.; Angunawela, I.; Zhang, J.; Qin, S.; Liebman-Pelaez, A.; Zhu, C.; Zhang, Z.; Ade, H.; Li, Y. HighPerformance All-Polymer Solar Cells: Synthesis of Polymer Acceptor by a Random Ternary Copolymerization Strategy. Angew. Chem., Int. Ed. 2020, 59, 15181-15185.

(31) Wang, W.; Wu, Q.; Sun, R.; Guo, J.; Wu, Y.; Shi, M.; Yang, W.; Li, H.; Min, J. Controlling Molecular Mass of Low-Band-Gap Polymer Acceptors for High-Performance All-Polymer Solar Cells. Joule 2020, 4, 1070-1086.

(32) Jia, T.; Zhang, J.; Zhong, W.; Liang, Y.; Zhang, K.; Dong, S.; Ying, L.; Liu, F.; Wang, X.; Huang, F.; Cao, Y. $14.4 \%$ efficiency allpolymer solar cell with broad absorption and low energy loss enabled by a novel polymer acceptor. Nano Energy 2020, 72, 104718.

(33) Sun, H.; Yu, H.; Shi, Y.; Yu, J.; Peng, Z.; Zhang, X.; Liu, B.; Wang, J.; Singh, R.; Lee, J.; Li, Y.; Wei, Z.; Liao, Q.; Kan, Z.; Ye, L.; Yan, H.; Gao, F.; Guo, X. A Narrow-Bandgap n-Type Polymer with an Acceptor-Acceptor Backbone Enabling Efficient All-Polymer Solar Cells. Adv. Mater. 2020, 32, 2004183.

(34) Fan, Q.; An, Q.; Lin, Y.; Xia, Y.; Li, Q.; Zhang, M.; Su, W.; Peng, W.; Zhang, C.; Liu, F.; Hou, L.; Zhu, W.; Yu, D.; Xiao, M.; Moons, E.; Zhang, F.; Anthopoulos, T. D.; Inganas, O.; Wang, E. Over $14 \%$ efficiency all-polymer solar cells enabled by a low bandgap polymer acceptor with low energy loss and efficient charge separation. Energy Environ. Sci. 2020, 13, 5017-5027.

(35) Luo, Z.; Liu, T.; Ma, R.; Xiao, Y.; Zhan, L.; Zhang, G.; Sun, H.; Ni, F.; Chai, G.; Wang, J.; Zhong, C.; Zou, Y.; Guo, X.; Lu, X.; Chen, H.; Yan, H.; Yang, C. Precisely Controlling the Position of Bromine on the End Group Enables Well-Regular Polymer Acceptors for AllPolymer Solar Cells with Efficiencies over 15\%. Adv. Mater. 2020, 32, 2005942.

(36) Peng, F.; An, K.; Zhong, W.; Li, Z.; Ying, L.; Li, N.; Huang, Z.; Zhu, C.; Fan, B.; Huang, F.; Cao, Y. A Universal Fluorinated Polymer Acceptor Enables All-Polymer Solar Cells with $>15 \%$ Efficiency. ACS Energy Lett. 2020, 5, 3702-3707.

(37) Fan, Q.; Su, W.; Chen, S.; Liu, T.; Zhuang, W.; Ma, R.; Wen, X.; Yin, Z.; Luo, Z.; Guo, X.; Hou, L.; Moth-Poulsen, K.; Li, Y.; Zhang, Z.; Yang, C.; Yu, D.; Yan, H.; Zhang, M.; Wang, E. A NonConjugated Polymer Acceptor for Efficient and Thermally Stable AllPolymer Solar Cells. Angew. Chem., Int. Ed. 2020, 59, 19835-19840.

(38) Dang, D.; Yu, D.; Wang, E. Conjugated Donor-Acceptor Terpolymers Toward High-Efficiency Polymer Solar Cells. Adv. Mater. 2019, 31, 1807019.

(39) Chen, D.; Yao, J.; Chen, L.; Yin, J.; Lv, R.; Huang, B.; Liu, S.; Zhang, Z.-G.; Yang, C.; Chen, Y.; Li, Y. Dye-Incorporated Polynaphthalenediimide Acceptor for Additive-Free High-Perform- ance All-Polymer Solar Cells. Angew. Chem., Int. Ed. 2018, 57, 45804584.

(40) Liu, X.; Zhang, C.; Duan, C.; Li, M.; Hu, Z.; Wang, J.; Liu, F.; Li, N.; Brabec, C. J.; Janssen, R. A. J.; Bazan, G. C.; Huang, F.; Cao, Y. Morphology Optimization via Side Chain Engineering Enables AllPolymer Solar Cells with Excellent Fill Factor and Stability. J. Am. Chem. Soc. 2018, 140, 8934-8943.

(41) Wu, Y.; Schneider, S.; Walter, C.; Chowdhury, A. H.; Bahrami, B.; Wu, H.-C.; Qiao, Q.; Toney, M. F.; Bao, Z. Fine-Tuning Semiconducting Polymer Self-Aggregation and Crystallinity Enables Optimal Morphology and High-Performance Printed All-Polymer Solar Cells. J. Am. Chem. Soc. 2020, 142, 392-406.

(42) Kolhe, N. B.; Tran, D. K.; Lee, H.; Kuzuhara, D.; Yoshimoto, N.; Koganezawa, T.; Jenekhe, S. A. New Random Copolymer Acceptors Enable Additive-Free Processing of $10.1 \%$ Efficient AllPolymer Solar Cells with Near-Unity Internal Quantum Efficiency. ACS Energy Lett. 2019, 4, 1162-1170.

(43) Liu, S.; Song, X.; Thomas, S.; Kan, Z.; Cruciani, F.; Laquai, F.; Bredas, J.-L.; Beaujuge, P. M. Thieno[3,4-c]Pyrrole-4,6-Dione-Based Polymer Acceptors for High Open-Circuit Voltage All-Polymer Solar Cells. Adv. Energy Mater. 2017, 7, 1602574.

(44) Lee, J.-W.; Sung, M. J.; Kim, D.; Lee, S.; You, H.; Kim, F. S.; Kim, Y.-H.; Kim, B. J.; Kwon, S.-K. Naphthalene Diimide-Based Terpolymers with Controlled Crystalline Properties for Producing High Electron Mobility and Optimal Blend Morphology in AllPolymer Solar Cells. Chem. Mater. 2020, 32, 2572-2582.

(45) Li, Z.; Xu, X.; Zhang, W.; Meng, X.; Ma, W.; Yartsev, A.; Inganäs, O.; Andersson, M. R.; Janssen, R. A. J.; Wang, E. High Performance All-Polymer Solar Cells by Synergistic Effects of FineTuned Crystallinity and Solvent Annealing. J. Am. Chem. Soc. 2016, 138, 10935-10944.

(46) Sun, H.; Liu, B.; Koh, C. W.; Zhang, Y.; Chen, J.; Wang, Y.; Chen, P.; Tu, B.; Su, M.; Wang, H.; Tang, Y.; Shi, Y.; Woo, H. Y.; Guo, X. Imide-Functionalized Heteroarene-Based n-Type Terpolymers Incorporating Intramolecular Noncovalent Sulfur..Oxygen Interactions for Additive-Free All-Polymer Solar Cells. Adv. Funct. Mater. 2019, 29, 1903970.

(47) Li, H.; Zhao, Y.; Fang, J.; Zhu, X.; Xia, B.; Lu, K.; Wang, Z.; Zhang, J.; Guo, X.; Wei, Z. Improve the Performance of the All-SmallMolecule Nonfullerene Organic Solar Cells through Enhancing the Crystallinity of Acceptors. Adv. Energy Mater. 2018, 8, 1702377.

(48) Li, Y.; Zheng, N.; Yu, L.; Wen, S.; Gao, C.; Sun, M.; Yang, R. A Simple Phenyl Group Introduced at the Tail of Alkyl Side Chains of Small Molecular Acceptors: New Strategy to Balance the Crystallinity of Acceptors and Miscibility of Bulk Heterojunction Enabling Highly Efficient Organic Solar Cells. Adv. Mater. 2019, 31, 1807832.

(49) Zhang, M.; Guo, X.; Ma, W.; Ade, H.; Hou, J. A Large-Bandgap Conjugated Polymer for Versatile Photovoltaic Applications with High Performance. Adv. Mater. 2015, 27, 4655-4660.

(50) Fan, Q.; Xu, Z.; Guo, X.; Meng, X.; Li, W.; Su, W.; Ou, X.; Ma, W.; Zhang, M.; Li, Y. High-Performance Nonfullerene Polymer Solar Cells with Open-Circuit Voltage over $1 \mathrm{~V}$ and Energy Loss as Low as $0.54 \mathrm{eV}$. Nano Energy 2017, 40, 20-26.

(51) Su, W.; Fan, Q.; Guo, X.; Meng, X.; Bi, Z.; Ma, W.; Zhang, M.; Li, Y. Two Compatible Nonfullerene Acceptors with Similar Structures as Alloy for Efficient Ternary Polymer Solar Cells. Nano Energy 2017, 38, 510-517.

(52) Hou, J.; Inganäs, O.; Friend, R. H.; Gao, F. Organic solar cells based on non-fullerene acceptors. Nat. Mater. 2018, 17, 119-128. 